\title{
sciendo
}

\section{Acute and Delayed Effects of Fatigue on Ground Reaction Force, Lower Limb Stiffness and Coordination Asymmetries During a Landing Task}

\author{
by \\ Débora Aparecida Knihs' ${ }^{1}$, Haiko Bruno Zimmermann ${ }^{2}$, Juliano Dal Pupo ${ }^{1}$
}

Landing is a critical phase of movement for injury occurrence, in which lower limbs should be used equally to better absorb the shock. However, it has been suggested that fatigue can lead to the appearance of asymmetries. The aim of this study was to verify the acute and delayed effects of fatigue on the lower limb asymmetry indexes of peak ground reaction force, leg stiffness and intra-limb coordination during a landing task. Fifteen physically active men performed a fatigue protocol composed of 14 sets of 10 continuous vertical jumps, with a one-minute rest interval between the sets. A step-off landing task was performed before, immediately after, $24 \mathrm{~h}$ and $48 \mathrm{~h}$ after the fatigue protocol. Two force plates and a video analysis system were used. The symmetry index equation provided the asymmetry indexes. For statistical analysis, ANOVA and effect size analysis were utilized. Inferential statistics did not show the effect of fatigue in the asymmetry indexes for any variable or condition $(p>.05)$. Moderate effect sizes were observed for peak ground reaction force (0.61) and leg stiffness (0.61) immediately after the application of the protocol. In conclusion, fatigue does not seem to significantly change the asymmetries of lower limbs, especially regarding intra-limb coordination. The moderate effects observed for peak ground reaction force and leg stiffness asymmetries suggest that these variables may be acutely affected by fatigue.

Key words: stretch-shortening cycle, shock absorption, spring-mass model, continuous relative phase.

\section{Introduction}

The biomechanics of landing in vertical jumps and sports movements in general has been frequently studied mainly due to its association with lower limb injuries (Aerts et al., 2013; Dufek and Bates, 1991; Wang, 2009; Wong et al., 2020). The landing is a phase of movement with high impact, in which the majority of non-contact injuries are suggested to occur (Aerts et al., 2013; James et al., 2006; Hughes and Watkins, 2008). During landings large ground reaction force loads are experienced (Hovey et al., 2019; Wang, 2009) which, added to small movement excursions (Hovey et al., 2019), may contribute to the increased risk of lower limb injuries, such as stress fracture and anterior cruciate ligament rupture (Aerts et al., 2013).

During bilateral tasks, such as landings, the movement requires good coordination and should occur symmetrically. This would allow for an equal distribution of the load between the limbs, better shock absorption upon landing and forces acting upon the system (Artes et al., 2013), minimizing the number of injuries (Dufek and Bates, 1991). It is known that some level of between limbs asymmetry (i.e., the difference between one limb and the other) is present in a variety of variables during landing tasks (Dufek and Bates, 1991; Hughes and Watkins, 2008).

\footnotetext{
1 - Federal University of Santa Catarina, Biomechanics Laboratory, Florianopolis - Brazil.

2 - Regional University of Blumenau, Physical Education Department, Blumenau - Brazil.
} 
However, it has been suggested that pronounced asymmetry may indicate a predisposition to lower limb injuries (Bishop et al., 2018b; Exell et al., 2016; Impellizzeri et al., 2010; Maloney, 2018) as well as it seems to affect sports performance (Bell et al., 2014).

Recent studies have suggested that fatigue is a possible factor that influences lower limb asymmetry (Bishop et al., 2018a; Radzak et al., 2017; Zifchock et al., 2008). In general, muscle fatigue mainly from exhaustive stretch-shortening cycles (SSC) causes changes in muscle function, leading to impairment of neuromuscular performance (e.g., loss of strength and muscle power) (Nicol et al., 2006). Consequently, it may affect variables related to movement control as ground reaction force, lower limb stiffness and lower limb coordination (Dal Pupo et al., 2013; James et al., 2006; Kuitunen et al., 2007). These variables are important in regulating shock absorption through the lower limbs during the landing (Santello, 2005), which is a common task in different sports. In this line, Wong et al. (2020) recently verified that fatigue caused changes in the knee range of motion and in the energy absorption ability during the landing phase of a forward drop jump to a vertical jump. It is suggested that fatigue has the potential to cause, accentuate (Radzak et al., 2017; Zifchock et al., 2008) or even decrease (Carpes et al., 2007; Radzak et al., 2017) asymmetries between the lower limbs, due to the appearance of irregular patterns in the movement technique and limb dominance. Traditionally, asymmetries between legs are analyzed using strength variables (Bishop et al., 2018b; Maloney, 2018); however, motor control variables such as stiffness and coordination need to be explored to help understand the changes or adaptations of the landing movement under fatigue and its possible links to shock absorption and potential risk of injuries.

Additionally, fatigue from exercises involving the SSC, such as vertical jumps, has a complex and versatile response (Byrne et al., 2004; Kuitunen et al., 2007; Nicol et al., 2006), and its effects on neuromuscular and proprioceptive variables may last for days (Nicol et al., 2006). However, to our knowledge, there is no evidence of the delayed effects of fatigue on asymmetries, especially related to motor control variables such as leg stiffness and intra-limb coordination during landings.

Thus, the aim of this study was to investigate the acute and delayed effects of a SSC muscle fatigue on the indexes of asymmetry of ground reaction force, leg stiffness and intra-limb coordination during a landing task. The main hypothesis was that the indexes of asymmetry for all variables would increase (i.e., making the individuals more asymmetric) immediately after the fatigue protocol.

\section{Methods}

\section{Participants}

Fifteen males (age: $26.9 \pm 3.3$ years, body mass: $80.0 \pm 11.7 \mathrm{~kg}$, body height: $181.7 \pm 6.8 \mathrm{~cm}$, body fat percentage: $13.1 \pm 4.3 \%$ ) participated in this study. The sample size was defined a priori (GPOWER $^{\circledR}$ software) given $\alpha=0.05$, expected power of 0.8 and moderate effect size (0.5). A minimum sample size of 15 individuals was obtained, although we recruited 17 athletes to account for possible sample loss. Participants were physically active men who practiced physical exercises (strength training, running and/or sports involving jumps) three to five times a week, for at least one year and had no injuries or pathologies that could compromise their ability to perform maximum effort during the tests. They were requested to abstain from physical exercises involving the lower limbs $24 \mathrm{~h}$ prior to testing. Two individuals were excluded from the study because they violated this criteria. All the procedures were conducted according to the Declaration of Helsinki, and before the data collection participants signed a consent form approved by the local ethics committee that guaranteed their rights and anonymity (CAAE= 87443318.9.0000.0121).

\section{Study design}

This is an experimental design study. Participants performed a fatigue protocol composed of 14 sets of 10 continuous vertical jumps (independent variable). Before, immediately after, $24 \mathrm{~h}$ and $48 \mathrm{~h}$ after the fatigue protocol, participants performed three trials of the step-off landing (STL) task, using two force plates and a video analysis system. Dependent variables measured during the STL task were peak ground reaction force, leg stiffness and a continuous relative phase, measured for both legs to calculate the asymmetry indexes. 


\section{Procedures}

All testing procedures took place in a biomechanics laboratory. Participants were asked to wear the same pair of sneakers on all testing days, to prevent the use of varied shoes from having an effect on the variables analyzed (Wang et al., 2017). During the first visit, participants performed a familiarization session to learn and practice the step-off landing (STL) movement. This task was chosen because it provides a standardized drop height for all individuals and allows greater control of intervening factors (Edwards et al., 2010; Yeow et al., 2010; Zhang et al., 2008). Participants repeated the movement as many times as they needed until an evaluator judged the movement to be appropriate for participation in the study (without jumping-off the box, landing with both legs at the same time, good balance). The leg preference of participants was assessed in this day with the question: "what is your favorite leg to kick a ball with?". It was also requested to test which leg they preferred / felt more comfortable to take the first step forward from the box in the step-off landing movement. All participants preferred the right leg in both situations. Also on this day, the anthropometric measurements of participants were taken to assess fat content (stature, body mass, skinfolds) and kinematic processing (lengths and diameters of the lower limbs).

During the second session (at least $24 \mathrm{~h}$ apart) participants returned to the laboratory to conduct the experiment. First, the recovery level of all participants was evaluated through a rate of perceived recovery scale (Laurent et al., 2011), with magnitude from 0 to 10,0 being very poorly recovered and 10 very well recovered. Additionally, perceptual muscle soreness in some body parts (anterior thigh, posterior thigh and posterior leg) was assessed using a visual analogue scale (Price et al., 1983) ranging from ' 0 ' (no pain) to ' 10 ' (worst pain). Then, a warm-up consisting of 10 minutes on a cycloergometer at $90 \mathrm{~W}$ was performed. Reflective markers (14 $\mathrm{mm})$ for kinematic analysis were placed on both sides of the body on the following anatomical points: acromion, major trochanter, anterior superior iliac spine, posterior superior iliac spine, medial thigh, epicondyle lateral femoral, medial leg, lateral malleolus, calcaneus and proximal phalanx of the hallux. Afterwards, each participant performed the STL task three times before the fatigue protocol (i.e., baseline). Participants started the movement in the standing position, with hands on the hips, on the top of a box $45 \mathrm{~cm}$ in height. After a voice command, the participant took a step forward with the preferred leg, leaving the box without jumping, landing with both feet on two individual triaxial force plates (AMTI ${ }^{\circledR}$ OR6-7-OP-2000, USA), naturally returning to the orthostatic position. Three-dimensional kinematics were obtained during the STL task using a system with eight high-speed integrated cameras (VICON ${ }^{\circledR}, \mathrm{MX}$ systems, Oxford, UK), calibrated and synchronized with the force plates. Participants performed then the fatigue protocol (described below).

Immediately following the fatigue protocol, participants performed the STL task three more times to represent a post-fatigue condition. Participants returned to the laboratory $24 \mathrm{~h}$ and 48 $\mathrm{h}$ after the fatigue protocol to repeat the STL assessment, following the same procedures as described above. Participants were requested not to undergo physical training during the follow up days of assessment. The recovery level of participants was evaluated again through the rate of perceived recovery scale before assessments at $24 \mathrm{~h}$ and $48 \mathrm{~h}$.

Fatigue protocol

The fatigue protocol was composed of 14 sets of 10 continuous vertical jumps with oneminute intervals between sets. This protocol was based on a previous jumping protocol frequently used to induce SSC fatigue composed of 10 sets of 10 countermovement jumps (Lazaridis et al., 2018; Twist and Eston, 2005). Based on a pilot study, we slightly increased the number of sets (additional 4 sets) to achieve a higher fatigue index (i.e., at least $20 \%$ ) that was desired in the present study. The jumps were performed on a piezoelectric force plate (Kistler® Quattro Jump, 9290 AD, Switzerland) and participants were asked to maintain the hands on the hips, the trunk as vertical as possible and flex the knees approximately to $90^{\circ}$ in the descending phase of the jumps. Strong verbal encouragement was given to participants to perform the jumps with the maximum intensity possible.

At the end of the fatigue protocol, participants were asked to rate their perceived exertion using a scale (Foster et al., 2001) of magnitude $0-10(0=$ no effort $/$ rest; $10=$ maximal effort), referring to the total effort applied to 
perform the fatigue protocol. The fatigue index (Equation 1) was used to verify the percentage drop in jump performance during the protocol (Dal Pupo et al., 2013). Where: PMEAN_4J = average power of the first four jumps, and PMean_end4J = average power of the last four jumps. Power output was calculated by multiplying ground reaction force with the velocity of the centre of mass (obtained by integration of acceleration) during the propulsive phase of jumps.

$$
\text { Fatigue Index }=\frac{P_{\text {MEAN_4J }}-P_{\text {MEAN_END4J }}}{P_{\text {MEAN_4J }}} \times 100
$$

(Equation 1)

\section{Data analysis and dependent variables}

Ground reaction force (GRF) data were obtained during the STL task through the force plates. It was filtered using a low-pass, fourthorder Butterworth filter with a cut-off frequency of $10 \mathrm{~Hz}$, determined from spectral analysis. The maximum value of the vertical GRF curve during the initial ground contact phase was considered the peak of GRF (P $P_{\mathrm{GRF}}$ ).

Three-dimensional reconstruction of the STL task was realized through Nexus ${ }^{\circledR}$ software, and then the coordinates of the reflective markers were obtained and filtered using a fourth-order Butterworth low-pass filter with a cut-off frequency of $10 \mathrm{~Hz}$, determined from spectral analysis. In Matlab ${ }^{\circledR}$ software (2013a, The MathWorks Inc.) the segmental angles of the trunk, thigh and leg (formed between the respective segments and a horizontal plane) were determined for the intra-limb coordination analysis. The continuous relative phase (CRP) was calculated to assess the intra-limb coordination (Dal Pupo et al., 2013; Hamill et al., 1999). The CRP was analysed on both sides of the body for the trunk-thigh and thigh-leg couplings, in the descendent (i.e. the moment when the feet touched the force plates until the maximal knee flexion) and ascendant (i.e. the moment when knees started to extend until the natural vertical posture of the participant) phases of the STL task. The root mean square (RMS) of the temporal coordination curve was calculated to represent each phase.

Leg stiffness was obtained using the spring-mass model and calculated as the ratio between the PGRF and the change in the length of the lower limb (Serpell et al., 2012) during landing.
Using three-dimensional reconstruction (Nexus ${ }^{\circledR}$ ) and mathematic routines (Matlab ${ }^{\circledR}, 2013 a$, The MathWorks Inc.), the length of the lower limb (distance between the hip and the ankle) was identified in the vertical position and in the position of greatest flexion in the ground contact phase, and through this, the change in the length of the lower limb was obtained. Leg stiffness was measured for each lower limb individually.

The Symmetry Index equation (high value - lower value / total value $x 100$ ) was chosen for the calculation of between limbs asymmetrical magnitude (Shorter et al., 2008). This is one of the most indicative equations when analyzing bilateral tasks, showing great accuracy (Bishop et al., 2018a). This equation always provided positive values, used for absolute calculation and analysis. Additionally, for an individual analysis of the direction of asymmetries (i.e. the side on which the asymmetry appears), we added a minus sign to the values where the left limb showed a greater value. Statistical analysis

Initially, the descriptive analysis of the data was performed. One-way repeated measure analysis of variance (ANOVA) was used to compare the indexes of asymmetry over the time (pre, post, 24 and $48 \mathrm{~h}$ ), with Sidak post-hoc performed when necessary. Data sphericity was tested (Mauchly's Test) and corrected (Greenhouse Geiser) when necessary. The level of significance was set at $p \leq 0.05$. As an additional analysis, the magnitude of the differences [(Mean 1 - Mean 2) / SD pooled)] was verified through effect sizes (ES - Hedge) and the results were classified as $<0.2$ trivial; 0.2 - 0.6 small; 0.6 - 1.2 moderate; 1.2 - 2.0 large; 2.0 - 4 very large; and $>4.0$ near perfect (Hopkins et al., 2009). Statistical procedures were performed using SPSS ${ }^{\circledR}$ (Statistical Package for Social Sciences) v.17.0 (SPSS Inc., USA) software.

\section{Results}

The fatigue index and the rate of perception of the protocol were $25.3 \pm 11.4 \%$ and $7.6 \pm 1.5$, respectively.

ANOVA did not show any effects in the indexes of asymmetry of $\mathrm{P}_{\mathrm{GRF}}(\mathrm{F}=2.67, p=.091$, Figure 1a) as well as leg stiffness $(\mathrm{F}=2.01, p=.126$, Figure $1 b)$. No effect was also observed for the asymmetry of the trunk-thigh CPR, neither in the descendent phase ( $\mathrm{F}=1.01, p=.397$, Figure 2a), nor the ascendent phase $(\mathrm{F}=0.41, p=.745$, Figure $2 \mathrm{~b})$ as well as for the asymmetry of the thigh-leg CPR in 
the descendent phase $(\mathrm{F}=0.91, p=.448$, Figure 2c) and the ascendent phase $(\mathrm{F}=0.83, p=.429$, Figure 2d) of the landing task.

Analysis of the effect sizes was conducted to compare post, $24 \mathrm{~h}$ and $48 \mathrm{~h}$ conditions with the baseline (Table 1). Moderate effect sizes were observed for $\mathrm{P}_{\mathrm{GRF}}(+58.2 \%)$ and leg stiffness $(+60.9 \%)$ immediately after (post) the application of the fatigue protocol, suggesting an increase in these variables. No other relevant effect (ES $\geq$ moderate) was found for these variables at $24 \mathrm{~h}$ and $48 \mathrm{~h}$ (i.e., no delayed effects). The CPR indexes of asymmetry of trunk-thigh and thigh-leg couplings did not present important changes (ES $\geq$ moderate) at any time in any phase of movement.

Table 1

Effect sizes of asymmetries when compared to pre-condition

\begin{tabular}{lccc}
\hline & Pre vs. Post & Pre vs. 24h & Pre vs. 48h \\
& ES (CI 95 \%) & ES (CI 95 \%) & ES (CI 95 \%) \\
\hline \multirow{2}{*}{ PGRF } & $\mathbf{0 . 6 1}$ & 0.11 & 0.32 \\
& $(0.23-0.99)$ & $(-0.43-0.66)$ & $(-0.18-0.83)$ \\
Leg Stiffness & $\mathbf{0 . 6 1}$ & 0.12 & 0.24 \\
& $(0.09-1.13)$ & $(-0.48-0.73)$ & $(-0.32-0.79)$ \\
CRP Trunk-Thigh - DP & 0.32 & 0.26 & 0.51 \\
& $(-0.35-1.00)$ & $(-0.33-0.87)$ & $(-0.15-1.23)$ \\
CRP Trunk-Thigh - AP & 0.02 & 0.22 & 0.30 \\
& $(-0.95-0.91)$ & $(-0.48-0.93)$ & $(-0.38-0.87)$ \\
CRP Thigh-Leg - DP & 0.37 & 0.02 & 0.55 \\
& $(-0.34-1.05)$ & $(-0.78-0,83)$ & $(-0.06-1.28)$ \\
CRP Thigh-Leg- AP & 0.53 & 0.26 & 0.32 \\
& $(-0.30-1.25)$ & $(-0.49-1.01)$ & $(-0.52-1.16)$ \\
\hline
\end{tabular}

Note: $E S=$ effect size; $C I=$ confidence interval; $C R P$ : Continuous Relative Phase; $D P=$ descendent phase; $A P=$ ascendent phase.

a)

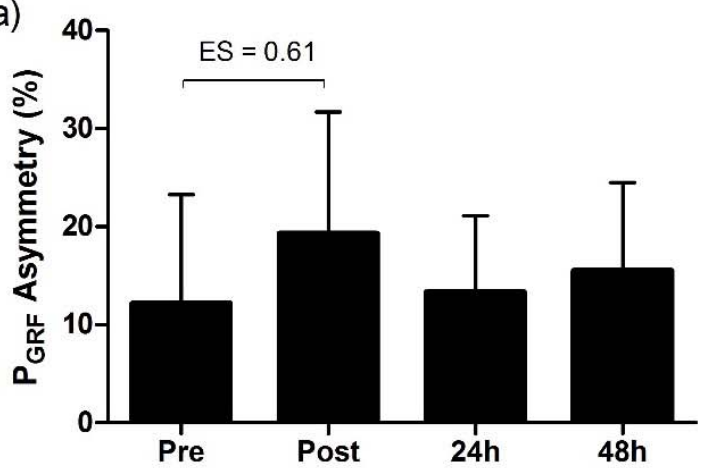

b)

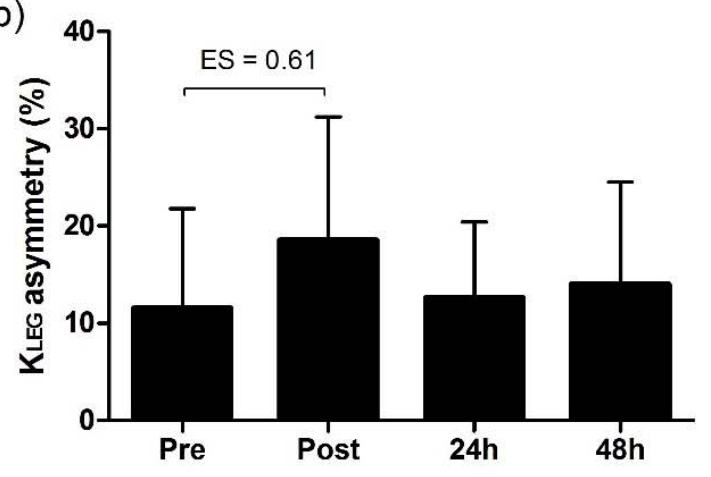

Figure 1

Peak of ground reaction force $\left(P_{G R F}\right)(a)$ and leg stiffness $\left(K_{L E G}\right)(b)$ indexes of asymmetry over time. Note: $E S=$ Effect size 
a) Trunk-Thigh

a.1)

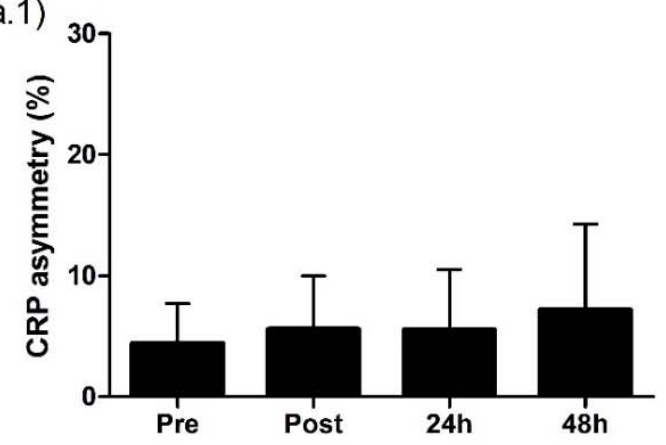

a.2)

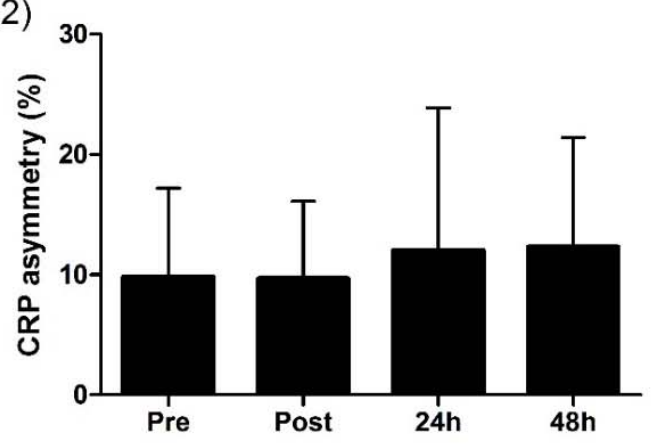

b) Thigh-Leg

b.1)

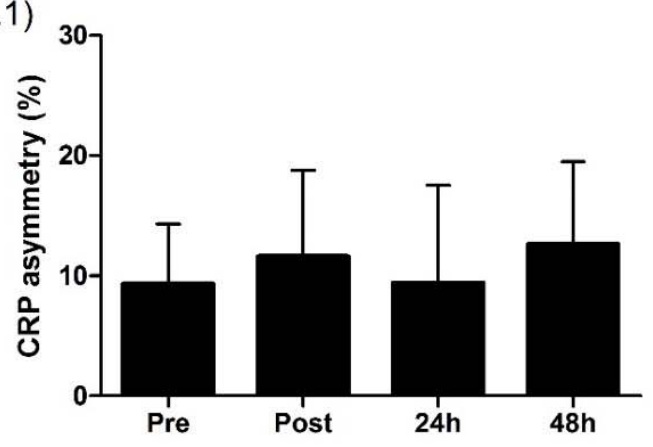

b.2)

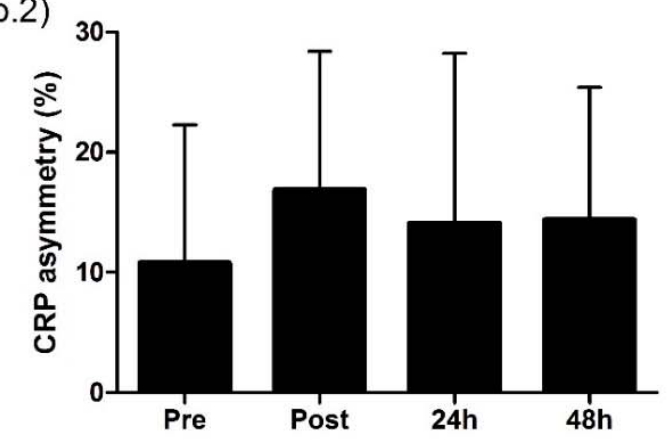

Figure 2

Continuous relative phase (CPR) indexes of asymmetry of trunk-thigh (a) and thigh-leg (b) couplings during the descendent (1) and ascendent (2) phases of movement, over time.

\section{Discussion}

The aim of this study was to verify the acute and delayed SSC fatigue effects on the indexes of asymmetry of peak ground reaction force, leg stiffness and intra-limb coordination during a landing task. Inferential statistics indicates that SSC fatigue does not seem to change the asymmetries of ground impact, stiffness and coordination of lower limbs during a landing task. However, the effect size analysis suggests that the $P_{\text {GRF }}$ and leg stiffness may be moderately acutely affected by fatigue.
Although non-significant, the moderated acute effect on indexes of asymmetry (approximately $60 \%$ of change in the average in relation to the SD for the variables PGRF and leg stiffness) observed after fatigue suggests that the lower limbs were affected at different rates by fatigue (Carpes et al., 2007; Radzak et al., 2017). These differences generally emerge due to limb preference or dominance during motor tasks. Considering that fatigue decreases force production (Nicol et al., 2006), if one limb is more affected by fatigue, a misbalance of force between limbs may arise. It is interesting to observe that the asymmetric indexes 
of $P_{\text {GRF }}$ and leg stiffness reached values above 15\% immediately after the fatigue protocol. Asymmetries greater than $15 \%$ between the lower limbs in strength/force variables (as PGRF) have been suggested as a potential to predisposition to injury occurrence (Bishop et al., 2018b; Exell et al., 2016; Impellizzeri et al., 2007), probably because motor control variables which regulate the shock absorption through the lower limbs during the landing can be affected (Santello, 2005).

Previous studies found influence of fatigue on ground reaction force or stiffness asymmetry during free-weight barbell back squat exercise (Hodges et al., 2011) and sprint running (Girard et al., 2017; Radzak et al., 2017), but not during landing tasks, making the comparison of results difficult. In general, Radzak et al. (2017) observed that vertical stiffness became more symmetrical, and knee stiffness more asymmetrical under fatigue conditions, while Girard et al. (2017) did not verify alterations in stiffness asymmetries after repeated sprints and Hodges et al. (2011) did not verify changes in ground reaction force asymmetries after sets of squats. Stiffness is an important variable for shock absorption (Santello, 2005) and performance (Kuitunen et al., 2007; Radzak et al., 2017); thus to know that a possible asymmetric state of leg stiffness can be induced under fatigue conditions may have some practical implications.

Previous studies have found that fatigue may affect motor control variables in lower extremity coordination (Dal Pupo et al., 2013; James et al., 2006; Lazaridis et al., 2018). Additionally, it was suggested that fatigue could affect the legs differently (Bishop et al., 2018b; Radzak et al., 2017; Zifchock et al., 2008), promoting or accentuating asymmetries. Thus, we hypothesized that fatigue could increase coordination asymmetries between limbs. However, in the present study, intra-limb coordination asymmetry did not suffer any significant and important alteration due to fatigue, acute or delayed. To the authors 'knowledge this is the first study investigating the influence of fatigue on intra-limb coordination asymmetries. Rodacki et al. (2001) hypothesized the presence of a 'common drive', difficult to change, that guides muscle activation and for consequence, the coordination, maintaining the movement pattern even under fatigue conditions. Thus, it can be speculated that if the coordination of each lower limb cannot be easily changed, patterns of asymmetry in this variable also cannot emerge or be accentuated. In addition, another study which confirmed no effects of fatigue on asymmetry of kinetic and kinematic variables of running (Girard et al., 2017) suggests that both lower limbs get fatigued at similar rates.

It is known that SSC fatigue induces delayed effects on muscle function (Nicol et al., 2006). In the present study, the SSC fatigue protocol was able to produce a fatigue index of $25 \%$ (based on jump height decrement), but no delayed effects were visualized for any asymmetry variable. We can speculate that these delayed effects are mainly observed only for neuromuscular performance variables (e.g., muscle strength/power) (Byrne et al., 2004; Nicol et al., 2006). In addition, it is possible that the fatigue level generated by this protocol was not sufficient to alter, in a delayed condition, the index of asymmetry of the tested variables.

According to Bishop et al. (2018b), to investigate the fatigue effects on between-limb imbalances can help to understand the relationship of asymmetries with sports performance and injuries. The results of the present study provide new information on the topic (fatigue $x$ asymmetries) and add literature in the search for understanding behind these mechanisms. Although the results of inferential statistics in this study indicate that asymmetries of PGRF, stiffness and intra-limb coordination were not altered with fatigue, practical and non-inferential analysis suggests that $P_{G R F}$ and leg stiffness asymmetries may be moderately affected. Therefore, some attention should be paid by practitioners/athletes performing landing tasks under fatigue, such as at the end of volleyball or basketball matches and plyometric training. In these situations, important variables for shock absorption may became more asymmetrical, exposing the legs to different impacts, loads and stress, which may imply the development of neuromuscular imbalances and a possible risk of injury.

It is important to point that the present study has some limitations. The evaluations postfatigue were conducted with the shortest possible interval after the application of the protocol, however, the time between the completion of the exercise protocol and the evaluation was on 
average $80 \mathrm{~s}$. This may have allowed for some level of recovery, influencing the results. Also, care must be taken when transferring these results to practice, since the STL task does not replicate exactly the jump-landing task.

In conclusion, SSC fatigue does not seem to significantly change the lower limb indexes of asymmetry of variables related to motor control, especially intra-limb coordination; however, some moderate effects are suggested to be acute considering ground reaction force and leg stiffness asymmetries.

\section{Acknowledgements}

We would like to thanks the Coordenação de Aperfeiçoamento de Pessoal de Nível Superior (CAPES/PROEX), for the scholarship provided to the first author while conducting this project.

\section{References}

Aerts I, Cumps E, Verhagen E, Verschueren J, Meeusen R. A systematic review of different jump-landing variables in relation to injuries. J Sports Med Phys Fitness, 2013; 53(5): 509-519

Bell DR, Sanfilippo JL, Binkley N, Heiderscheit BC. Lean mass asymmetry influences force and power asymmetry during jumping in collegiate athletes. J Strength Cond Res, 2014; 28(4): 884-891

Bishop C, Read P, Lake J, Chavda S, Turner A. Inter-limb asymmetries: understanding how to calculate differences from bilateral and unilateral tests. Strength Cond J, 2018a; 40(4): 1-6

Bishop C, Turner A, Read P. Effects of inter-limb asymmetries on physical and sports performance: a systematic review. J Sports Sci, 2018b; 36(10): 1135-1144

Byrne C, Twist C, Eston R. Neuromuscular function after exercise-induced muscle damage theoretical and applied implications. Sports Med, 2004; 34(1): 49-69

Carpes FP, Rossato M, Faria IE, Bolli Mota C. Bilateral pedaling asymmetry during a simulated 40-km cycling time-trial. J Sports Med Phys Fitness, 2007; 47(1): 51-57

Dal Pupo J, Dias JA, Gheller RG, Detanico D, Santos SG. Stiffness, intralimb coordination, and joint modulation during a continuous vertical jump test. Sports Biomech, 2013; 12(3): 259-271

Dufek JS, Bates BT. Biomechanical factors associated with injury during landing in jump sports. Sports Med, 1991; 12(5): 326-337

Edwards S, Steele JR, McGhee DE. Does a drop landing represent a whole skill landing and is this moderated by fatigue? Scand J Med Sci Spor, 2010; 20(3): 516-523

Exell T, Irwin G, Gittoes M, Kerwin D. Strength and performance asymmetry during maximal velocity sprint running. Scand J Med Sci Sports, 2016; 27(11): 1273-1282

Foster C, Florhaug JA, Franklin J, Gottschall L, Hrovatin LA, Parker S, Doleshal P, Dodge C. A new approach to monitoring exercise training. J Strength Cond Res, 2001; 15(1): 109-115

Girard O, Brocherie F, Morin J, Millet GP. Lower limb mechanical asymmetry during repeated treadmill sprints. Hum Mov Sci, 2017; 52: 203-2014

Hamill J, Van Emmerik REA, Heiderscheit BC, Li L. A dynamical systems approach to lower extremity running injuries. Clin Biomech, 1999; 14(5): 297-308

Hodges SJ, Patrick RJ, Reiser II RF. Effects of fatigue on bilateral ground reaction force asymmetries during the squat exercise. J Strength Cond Res, 2011; 25(11): 3107-3117

Hopkins WG, Marshall SW, Batterham AM, Hanin, J. Progressive statistics for studies in sports medicine and exercise science. Med Sci Sports Exerc, 2009; 41(1): 3-13

Hovey S, Wang H, Judge LW, Avedesian JM, Dickin DC. The effect of landing type on kinematics and kinetics during single-leg landings. Sports Biomech, 2019; 18: 1-17 [Epub ahead of print]

Hughes G, Watkins J. Lower limb coordination and stiffness during landing from volleyball block jumps. Res Sports Med, 2008; 16(2): 138-154

Impellizzeri FM, Rampinini E, Maffiuletti N, Marcora SM. A vertical jump force test for assessing bilateral strength asymmetry in athletes. Med Sci Sports Exerc, 2007; 39(11): 2044-2050

James RC, Dufek JS, Bates BT. Effects of stretch shortening cycle exercise fatigue on stress fracture injury risk during landing. Res $Q$ Exerc Sport, 2006; 77(1): 1-13 
Kuitunen S, Kyröläinen H, Avela J, Komi PV. Leg stiffness modulation during exhaustive stretch-shortening cycle exercise. Scand J Med Sci Sports, 2007; 17(1): 67-75

Laurent CM, Green JM, Bishop PA, Sjökvist J, Schumacker RE, Richardson MT, Curtner-Smith M. A practical approach to monitoring recovery: development of a perceived recovery status scale.J Strength Cond Res, 2011; 24(3): 620-628

Lazaridis S, Patikas DA, Bassa E, Tsatalas T, Hatzikotoulas K, Ftikas C, Kotzamanidis C. The acute effects of an intense stretch-shortening cycle fatigue protocol on the neuromechanical parameters of lower limbs in men and prepubescent boys. J Sports Sci, 2018; 36(2): 131-139

Maloney SJ. The relationship between asymmetry and athletic performance: a critical review. J Strength Cond Res, 2018; 33(9): 2579-2593

Nicol C, Avela J, Komi P. The stretch-shortening cycle a model to study naturally occurring neuromuscular fatigue. Sports Med, 2006; 36(11): 977-999

Price DD, McGrath PA, Rafii A, Buckingham B. The validation of visual analogue scales as ratio scale measures for chronic and experimental pain. Pain, 1983;17(1):45-56

Radzak KN, Putnam AM, Tamura K, Hetzler RK, Stickley CD. Asymmetry between lower limbs during rested and fatigued state running gait in healthy individuals. Gait Posture, 2017; 51: 268-274

Rodacki ALF, Fowler NE, Bennett SJ. Multi-segment coordination: Fatigue effects. Med Sci Sports Exerc, 2001; 33(7): 1157- 1167

Santello M. Review of motor control mechanisms underlying impact absorption from falls. Gait Posture, 2005; 21(1): 85-94

Serpell BG, Ball NB, Scarvell JM, Smith PN. A review of models of vertical, leg and knee stiffness in adults for running, jumping or hopping tasks. J Sports Sci, 2012; 30(13): 1347-1363

Shorter K, Polk J, Rosengren K, Hsaio-Wecksler E. A new approach to detecting asymmetries in gait. Clin Biomech, 2008; 23(4): 459-467

Twist C, Eston R. The effects of exercise-induced muscle damage on maximal intensity intermittent exercise performance. Eur J Appl Physiol, 2005; 94(5-6): 652-658

Wang L. Lower extremity stiffness modulation: Effect of impact load of a landing task from different drop heights. Int SportMed J, 2009; 10(4): 186-193

Wang $\mathrm{X}$, Zhang $\mathrm{S}, \mathrm{Fu} \mathrm{W}$. Changes in impact signals and muscle activity in response to different shoe and landing conditions. J Hum Kinet, 2017; 56(1): 5-18

Wong TL, Huang CF, Chen PC. Effects of lower extremity muscle fatigue on knee loading during a forward drop jump to a vertical jump in female athletes. J Hum Kinet, 2020; 72(1): 5-13

Yeow $\mathrm{CH}$., Lee PVS, Goh JCH. Sagittal knee joint kinematics and energetics in response to different landing heights and techniques. The Knee, 2010; 17(2): 127-131

Zhang S, Derrick TR., Evans W, Yu Y-J. Shock and impact reduction in moderate and strenuous landing activities. Sports Biomech, 2008; 7(2): 296-309

Zifchock RA, Davis I, Higginson J, McCaw S, Royer T. Side-to-side differences in overuse running injury susceptibility: A retrospective study. Hum Mov Sci, 2008; 27(6): 888-902

\section{Corresponding author:}

\section{Débora Aparecida Knihs}

Federal University of Santa Catarina - Sports Center, Biomechanics Laboratory,

Deputado Antônio Edu Vieira, Pantanal, 88040-900 / Florianopolis, Santa Catarina, Brazil.

Phone number: +55 047 99622-5202

E-mail: deboraknihs@gmail.com 